\title{
Atlas-builder software and the eNeuro atlas: resources for developmental biology and neuroscience
}

\author{
Ellie S. Heckscher ${ }^{1, \pi}$, Fuhui Long, ${ }^{2, \pi}$, Michael J. Layden ${ }^{1, *}$, Chein-Hui Chuang ${ }^{1}$, Laurina Manning ${ }^{1}$, \\ Jourdain Richart ${ }^{1}$, Joseph C. Pearson ${ }^{3}$, Stephen T. Crews ${ }^{3}$, Hanchuan Peng ${ }^{2, \pm}$, Eugene Myers ${ }^{2, \S}$ and \\ Chris Q. Doe ${ }^{1, * *}$
}

\begin{abstract}
A major limitation in understanding embryonic development is the lack of cell type-specific markers. Existing gene expression and marker atlases provide valuable tools, but they typically have one or more limitations: a lack of single-cell resolution; an inability to register multiple expression patterns to determine their precise relationship; an inability to be upgraded by users; an inability to compare novel patterns with the database patterns; and a lack of three-dimensional images. Here, we develop new 'atlas-builder' software that overcomes each of these limitations. A newly generated atlas is three-dimensional, allows the precise registration of an infinite number of cell type-specific markers, is searchable and is openended. Our software can be used to create an atlas of any tissue in any organism that contains stereotyped cell positions. We used the software to generate an 'eNeuro' atlas of the Drosophila embryonic CNS containing eight transcription factors that mark the major CNS cell types (motor neurons, glia, neurosecretory cells and interneurons). We found neuronal, but not glial, nuclei occupied stereotyped locations. We added 75 new Gal4 markers to the atlas to identify over $50 \%$ of all interneurons in the ventral CNS, and these lines allowed functional access to those interneurons for the first time. We expect the atlas-builder software to benefit a large proportion of the developmental biology community, and the eNeuro atlas to serve as a publicly accessible hub for integrating neuronal attributes - cell lineage, gene expression patterns, axon/dendrite projections, neurotransmitters - and linking them to individual neurons.
\end{abstract}

KEY WORDS: Gal4 lines, Atlas, Gene expression, Interneurons, Neuronal diversity

\section{INTRODUCTION}

To understand how embryos develop, it is essential to uniquely identify cells and cell populations. In addition, the recent advent of genetic tools for manipulating gene expression, expressing vital markers, and modifying/monitoring neuronal activity becomes more useful if the cells expressing these tools are uniquely identifiable. One approach for identifying distinct cell types in

${ }^{1}$ Institute of Neuroscience, Institute of Molecular Biology, Howard Hughes Medical Institute, University of Oregon, Eugene, OR 97403, USA. 'Janelia Farm Research Campus, Howard Hughes Medical Institute, Ashburn, VA 20147, USA. ${ }^{3}$ Program in Molecular Biology and Biophysics, Department of Biochemistry and Biophysics, University of North Carolina at Chapel Hill, Chapel Hill, NC 275995, USA.

*Present address: Whitney Laboratory for Marine Bioscience, University of Florida, Saint Augustine, FL 32080, USA. ҒPresent address: Allen Institute for Brain Science Seattle, WA 98103, USA. \$Present address: Max-Planck Institute for Molecular Cell Biology and Genetics, Pfotenhauerstrasse 108, Dresden 01307, Germany.

These authors contributed equally to this work

**Author for correspondence (cdoe@uoregon.edu)

Received 5 February 2014; Accepted 14 April 2014 developing embryos is the generation of gene expression atlases. For example, the Eurexpress and the Allen Brain Atlas document thousands of gene expression patterns in the mouse embryo and brain, respectively (Diez-Roux et al., 2011; Lein et al., 2007). Similarly, FlyBase has a database of gene expression patterns in the developing fly embryo (Tomancak et al., 2007), and cell typespecific atlases have been generated for different stages of the Drosophila CNS (Beckervordersandforth et al., 2008; Broadus et al., 1995; Ito et al., 1995; Landgraf et al., 1997; Rickert et al., 2011; Wheeler et al., 2006, 2009). Despite the tremendous utility of these and similar databases, they typically have one or more limitations: a lack of single-cell resolution; an inability to register multiple expression patterns to determine their precise relationship; an inability to be upgraded by users; an inability to compare novel patterns with the database patterns; and a lack of a three-dimensional (3D) representation of the patterns.

Here, we develop new 'atlas-builder' software that overcomes each of these limitations. Our software can be used to create an atlas of any tissue in any organism with stereotyped cell positions. Newlygenerated atlases are $3 \mathrm{D}$, allow the precise registration of an infinite number of markers, are searchable and are open-ended because additional markers can be added. The 'atlas-builder' software is a publicly available resource (http://www.vaa3d.org). As a proof of principle, we generated an atlas of the late embryonic Drosophila CNS. We populated the atlas with eight transcription factor markers that allowed us to identify the major cell types in the CNS [motor neurons (MNs), glia, neurosecretory cells and interneurons]. To document the value of the atlas-builder software, we next added 75 Gal4 lines that are expressed in different, small subsets of neurons (fewer than 10 per hemisegment). This dramatically increases the number of identified interneurons in the CNS (to over 50\%) and provides new tools for manipulating and monitoring neuronal activity. The resulting 'eNeuro atlas' is also a publicly available resource (http://www.doelab.org/eNeuro-Atlas.html).

\section{RESULTS}

\section{Generation and characterization of the atlas-builder software}

We illustrate the utility of the atlas-builder software, using the late Drosophila ventral CNS as reference tissue. To generate an atlas of the Drosophila CNS, it was essential to have a single reference marker that labeled neurons distributed in all regions of the CNS; this 'fiduciary' marker can be used as a landmark to add all subsequent markers to the atlas. The Even-skipped (Eve) transcription factor is detected in the nuclei of a well-characterized subset of neurons distributed in all regions of the CNS, thus making it an excellent candidate for a fiduciary landmark (Doe et al., 1988; Frasch et al., 1987; Fujioka et al., 1999). Here, we focus on the second thoracic, third thoracic and first abdominal (T2, T3, A1) segments of the stage 16 embryonic CNS. 
Each of these segments has a set of unpaired midline cells plus bilaterally symmetric left and right 'hemisegments'. We chose these three segments because they contain much of the known diversity in the ventral CNS: T2, T3 and A1 have important segment-specific differences; and A1 serves as a proxy for the closely related posterior A2-A7 segments (Schmid et al., 1999). We developed computational tools to map the position of Eve+ nuclei from multiple samples (see Fig. 1A-C). These tools generate a 'single-marker' atlas (supplementary material Data File S1) and a list of associated statistics (supplementary material Data File S2). Each neuron is represented by a sphere, the size and position of which represents the average size and position of all data collected on that neuron. We found some variability in neuron position between embryos. Importantly, however, the average position of an Eve+ neuron was highly reproducible with an average s.d. of up to 1.0 cell diameter in all three axes (Fig. 1D).

Next, we tested whether all CNS cell types have equally reproducible cell body positions. To identify distinct neural subtypes, we generated single-marker atlases for additional transcription factor (TF) patterns: phospho-Mothers against Dpp (pMad; all MNs), Bar (subset MNs), Dimmed (Dimm; most neurosecretory cells), Reversed polarity (Repo; glia), Apterous (Ap; subset interneurons, subset neurosecretory cells), Engrailed (En; subset interneurons, subset glia) and Hb9 (Exex - FlyBase; subset interneurons, subset MNs) (Fig. 2A-G; supplementary material Data File S3) (Benveniste et al., 1998; Bourgouin et al., 1992; Broihier and Skeath, 2002; Garces et al., 2006;
Layden et al., 2006; Lundgren et al., 1995; Mccabe et al., 2003; O'Connor, 2005; Odden et al., 2002; Park et al., 2004, 2008; Patel et al., 1989; Xiong et al., 1994). Notably, we built our atlas at a time point slightly before a subset of neuropeptide (Dimm+) neurons would begin to project out of the ventral nerve cord (VNC; Allan et al., 2003; Miguel-Aliaga, 2004), thus rendering our Dimm + neurons pMad-. We found that all neuronal markers had highly reproducible average nuclear positions (Fig. $2 \mathrm{H}$ ). In contrast, glia often had highly variable nuclear positions (Fig. $2 \mathrm{H}$ ), even though their cellular positions are predictable (Beckervordersandforth et al., 2008; Ito et al., 1995). We concluded that interneurons, MNs and neurosecretory cells have highly stereotyped nuclear positions. Importantly, the same steps can be used to create an atlas of any marker in any organism, as long as cell or nuclear positions are stereotyped.

\section{Single-marker atlases can be merged to identify marker co-expression}

A single-marker atlas, as we have generated for each of the eight TFs, is of limited usefulness. But if cell body positions are sufficiently stereotyped, we should be able to use Eve to register, or merge, all atlases into the same 3D volume. This would identify cells co-expressing two or more markers, as well as allow us to determine the percentage of cells in the CNS that express at least one marker (or 'coverage'). We created computational tools to merge atlases, and used these tools to generate a single 'TF atlas' displaying all eight TFs expressed in 714 cells (Fig. 3A; supplementary material Data File S4).
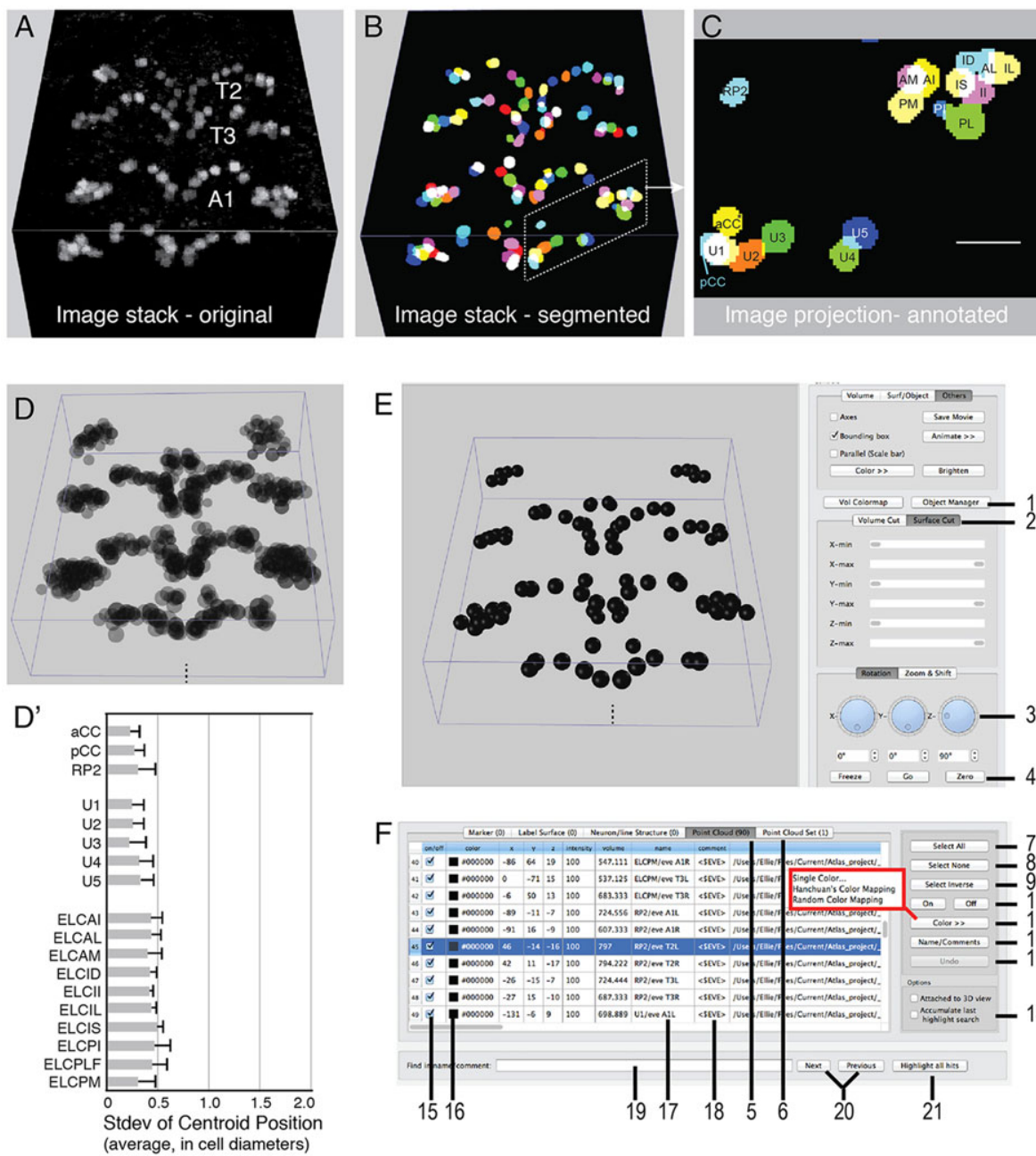
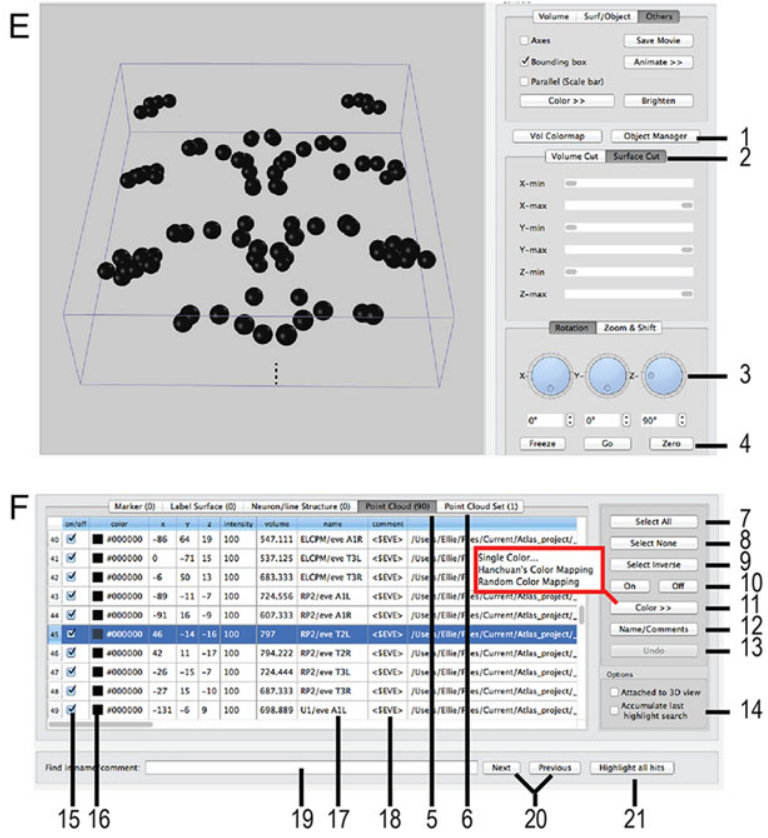

Fig. 1. Atlas building pipeline. (A) Image acquisition. A 3D rendering of a stage 16 CNS stained with anti-Even-skipped (Eve). Segments T2-A1 shown, anterior upwards, ventral view, rotated $45^{\circ}$ about anterior-posterior axis.

(B) Segmentation. Image from A after segmentation, with each nucleus as a distinct object and assigned a random color. (C) Annotation. Cells in A1R (white box in B). Each segmented object is given a user-defined name/marker prefix (e.g. U4/eve) and a hemisegment suffix (e.g. A1R). Anterior is towards the top, $z$-projection. Scale bar: $10 \mu \mathrm{m}$. (D) Registration. A 3D rendering of cells from nine segmented, annotated images. Stacks are overlaid to illustrate the variation in position between embryos for each Eve+ cell. ( $\left(^{\prime}\right)$ The average s.d. in cell body position for all Eve+ cells is a measure of the variance in position for each set of nuclei. $(E, F)$ User interface. In the final Eve single marker atlas, each nucleus is shown by a single sphere where diameter represents mean nuclear size and location represents mean $x, y, z$ position of each nucleus. A view of the Eve pattern in the atlas viewer was obtained by dragging and dropping the 'apo' file (supplementary material Data File S1) into the V3aaD window. See supplementary material Data File S9 for a description of functions 1-21. 

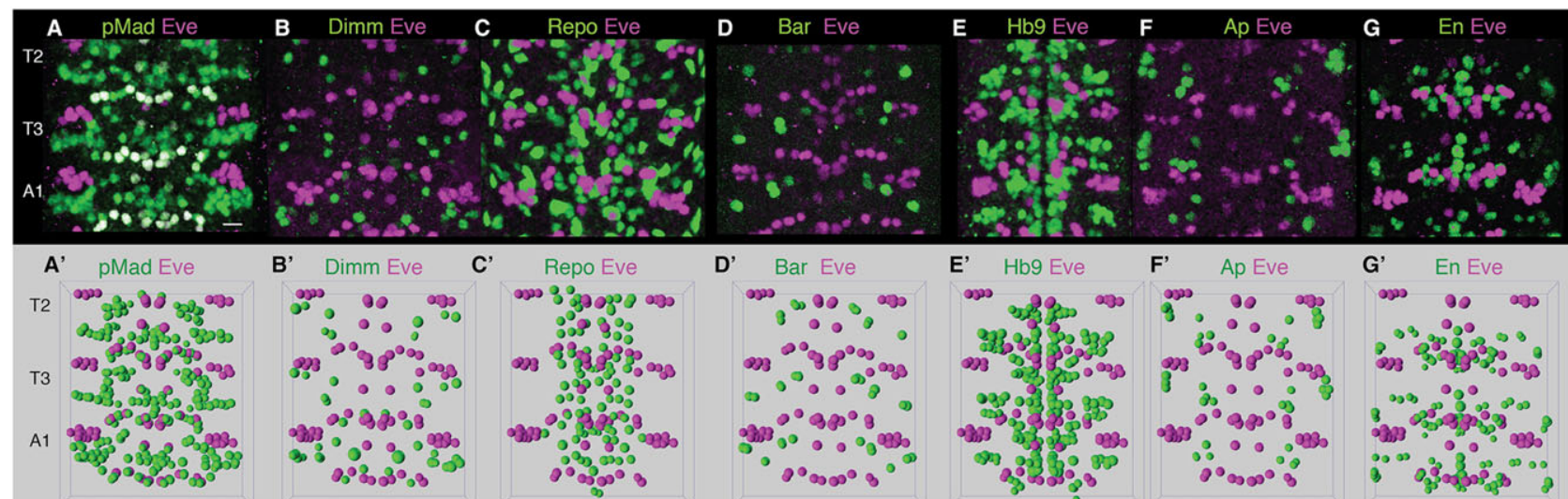

G' En Eve
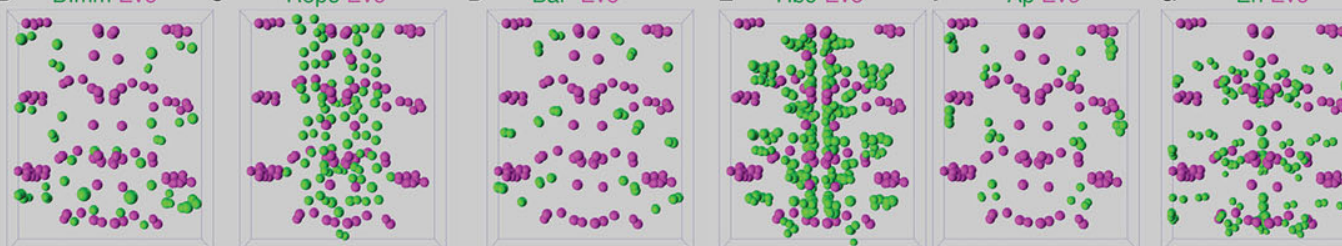

0200 $00 \quad 050$
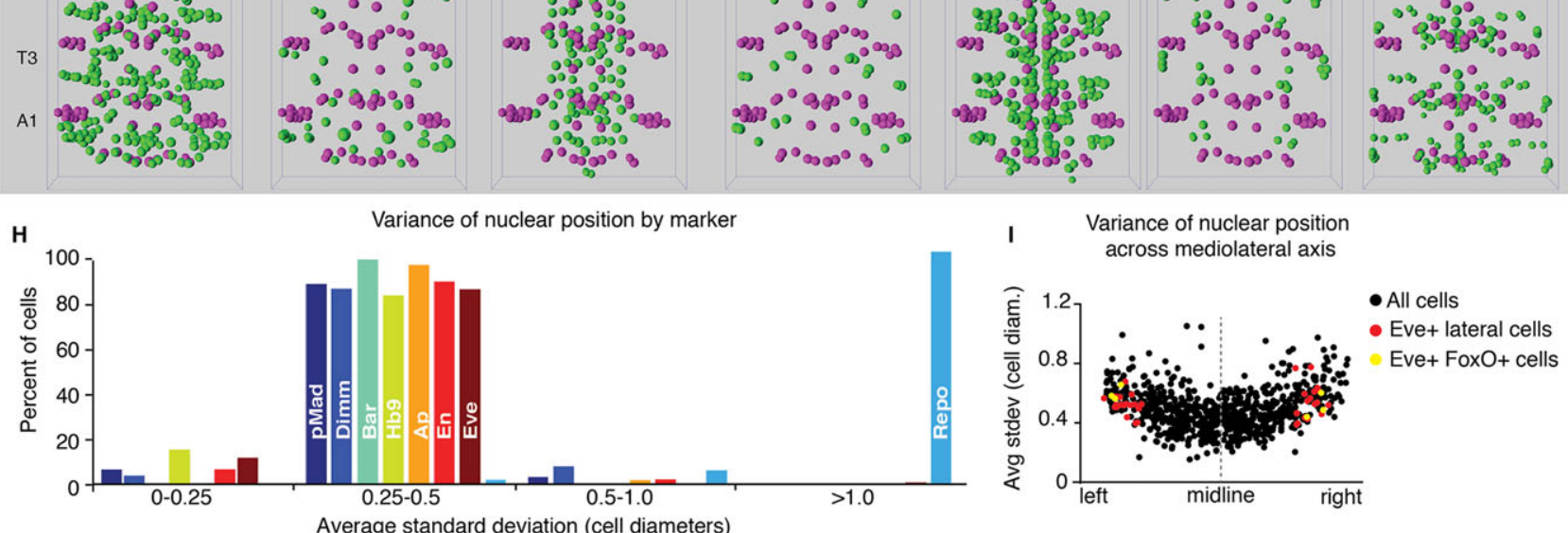

Average standard deviation (cell diameters)

Fig. 2. Cell bodies of neurons and a subset of glia have predictable positions in the embryonic CNS. (A-G) In vivo transcription factor expression patterns (green, names at top) co-stained with Even-skipped (Eve, magenta). Maximum intensity projections; co-expressing cells appear white. Scale bar: $10 \mu m$. FlyBase full names: pMad, phospho-Mothers against Dpp; Dimm, Dimmed; Repo, Reversed polarity; Bar, BarH1/BarH2; Hb9, Extra-extra; Ap, Apterous; En, Engrailed. ( $\left.A^{\prime}-G^{\prime}\right)$ Single-marker atlases for each pair of markers shown in A-G. Co-expressing cells are represented by overlapping green/magenta spheres, because single marker atlases do not account for co-expression. (See below for merged atlas showing co-expression.) $(\mathrm{H}) \mathrm{Histogram}$ showing the distribution of average s.d. of nuclear positions grouped by gene expression. Only glia (Repo+) have variable nuclear positions. (I) The average s.d. in nuclear position of the uniquely identifiable Eve+, FoxO+ neuron (yellow) is similar to that of the Eve+ lateral cluster of neurons (red) and of other laterally positioned neurons (black).

Importantly, the TF atlas merges overlapping cells that express two or more markers into a single cell.

To test the accuracy of the TF atlas - i.e. the number of false positives (two markers falsely predicted to co-express in a cell) or false negatives (two markers incorrectly assigned to separate cells when they are actually co-expressed) - we experimentally determined the actual overlap of all eight markers from our own stainings or from the literature (e.g. Fig. 3B,C). When we predicted co-expression solely based on proximity between cell body positions, we correctly identified $65 \%$ of all examples of in vivo co-expression (although the correct percentage rose to over $80 \%$ for neurons located in the medial CNS) (Fig. 3D; supplementary material Data File S5). When we added Rule 1, "if a pair of cells in one hemisegment is predicted to co-express a marker, then these pairs also co-express the marker in the other hemisegments", we increased correct predictions to $96 \%$, but this resulted in a high number of false positives (Fig. 3E). Ultimately, we settled on a set of conditions called Rule 2 that maximized true positives and negatives, while minimizing false positives and negatives (Fig. 3E; see Materials and Methods). Thus, we used the experimentally determined TF colocalization data as 'ground truth' to develop algorithms that allowed us to optimally determine whether two markers - stained in different embryos - are co-expressed or adjacent within the CNS.

\section{The eNeuro atlas: a single atlas containing eight transcription factors and $\mathbf{7 5}$ Gal4 lines}

We wanted to add a large number of new markers to generate a comprehensive eNeuro atlas. This would help us to validate our atlasbuilder software and to provide new markers for previously uncharacterized neurons (mostly interneurons). We chose to add Gal4 lines expressed in 1-10 neurons per hemisegment, which gave us the dual benefit of increasing known molecular markers for interneurons and of providing functional access to these neurons for the first time. From a previous large-scale screen of 5000 Gal4 lines (Manning et al., 2012), we selected 75 lines with strong, regular expression patterns at late embryonic stages. We used each Gal4 line to express a nuclearlocalized GFP, and created a single-marker atlas for each line (Fig. 4; supplementary material Data File S6). The 75 single marker atlases contained a total of 1998 cells (pre-merge) within the T2-A1 CNS, and $91 \%$ of the Gal4+ neurons had highly predictable positions (Fig. 4). We merged the Gal4 atlases with the TF atlas into a single, comprehensive 'eNeuro' atlas containing all 83 markers (supplementary material Data File S7). The eNeuro atlas contained fewer cells (1362) than the premerge numbers due to some neurons expressing multiple Gal4 lines, which were collapsed into a single neuron in the eNeuro atlas. Notably, we were able to experimentally validate $50 \%$ of the predicted co-expression in the eNeuro atlas (e.g. Fig. 5; supplementary material Data File S5). Thus, the eNeuro atlas integrated the co-expression of 83 molecular markers at single-cell resolution.

In the rodent spinal cord, over 20 different molecular subtypes of interneurons have been identified, but it is likely that interneuron diversity is much greater (Alaynick et al., 2011; Arber, 2012). Here, we used the eNeuro atlas to analyze the extent of interneuron diversity in the Drosophila embryonic ventral CNS. First, we assessed the coverage of the interneuron population. We defined interneurons as being negative for all motor neuron (pMad), neurosecretory (Dimm) and glial (Repo) markers (supplementary material Data File S8). The eNeuro atlas identified $59-60 \%$ of the interneuron population in 


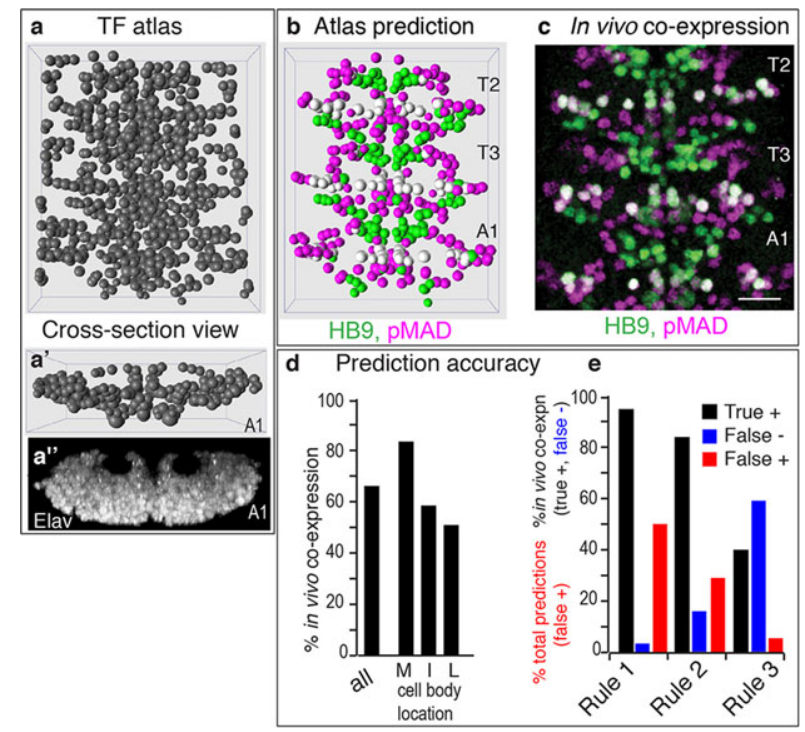

Fig. 3. Atlases can predict a majority of in vivo-determined co-expression. (A) The TF atlas is a merge of the eight TF single marker atlases. Anterior is upwards. $\left(A^{\prime}\right)$ Cross-section of neurons in $A 1$ of the TF atlas; clear zone is neuropil; dorsal is upwards. $\left(A^{\prime \prime}\right)$ The pan-neuronal marker Elav+ (Robinow and White, 1988) shows CNS morphology matching that of the atlas. Single optical cross-section ( $x z$ view) shown; clear zone is the neuropil; dorsal is upwards. Scale bar: $10 \mu \mathrm{m}$. (B) Atlas-predicted co-expression (white) for $\mathrm{Hb} 9$ (green) and pMad (magenta). Data used to build each atlas were from separate populations of embryos. (C) An embryo co-stained for Hb9 (green) and pMad (magenta) showing in vivo co-expression (white). Maximum intensity projection. Scale bar: $10 \mu \mathrm{m}$. (D) Scoring the accuracy of co-expression prediction based solely on distance between nuclei for co-expression among all TF single marker atlases ('all'), or for neurons with cell bodies located in the medial ('M'), intermediate ('l') or lateral ('L') one-third of the CNS. (E) Scoring the accuracy of co-expression prediction among all TF single-marker atlases, when additional assumptions are invoked (see Materials and Methods for definitions of rules). Assumptions can be changed to maximize true positives (Rule 1) or minimize false positives (Rule 3).

T2-A1 depending on the method of counting total neuron numbers (Fig. 6A,B). Analysis of the eNeuro atlas shows interneurons to be extremely diverse. We describe interneuron diversity for $\mathrm{A} 1$, but similar results were observed in thoracic hemisegments (Fig. 7; supplementary material Table S1 and Data File S8). The interneurons in A1 can be subdivided into 80 molecularly distinct subtypes, where a subtype is defined by a unique combination of markers (Fig. 7A,C). Furthermore, 43 combinations of markers label a unique single interneuron (Fig. 7B,C). Most of the molecular subtypes found in A1 were also found in other segments (Fig. 7D). Some molecular subtypes were found only in A1, and some were unique to thoracic segments (Fig. 7D). This indicates that at different anterior-posterior positions along the nerve cord there are molecularly distinct interneuron subtypes. Thus, addition of new markers to the eNeuro atlas, which is possible when using our atlas-builder software (see Discussion), should rapidly increase the number of molecularly distinct interneurons. We conclude that interneurons are characterized by extreme molecular diversity, and predict that each interneuron will have a unique, reproducible, transcriptional profile.

\section{The eNeuro atlas accurately identifies all known MNs and midline cells, and provides new genetic tools to characterize neuronal diversity}

Although our primary focus was on interneurons, we also used the eNeuro atlas to analyze midline cells and MNs. These populations are well characterized, which helps us to determine the accuracy of the eNeuro atlas. Midline cells contain the progeny of single-minded $(\mathrm{sim})+$ precursors, dorsal median cells and channel glia (Chiang et al., 1994; Ito et al., 1995; Kearney et al., 2004; Wheeler et al., 2006). We found that the position of midline cells in the eNeuro atlas strongly resembled the in vivo position of the midline cells (Fig. 8A,B; supplementary material Table S2 and Data File S8). Furthermore, we identified five Gal4 lines labeling subsets of midline cells that had not previously been distinguished at the molecular level (Fig. 8C; supplementary material Table S2). MNs include a set of 35 somatic MNs per hemisegment that can be molecularly subdivided based on Eve, $\mathrm{Hb} 9$ and Bar expression as well as visceral MNs (Landgraf and Thor, 2006; Miguel-Aliaga et al., 2008). The eNeuro atlas identified all Eve+, Hb9+ or Bar+ somatic MNs (Fig. 8D), and a group of poorly characterized Eve/ Hb9/Bar triple-negative MNs (Fig. 8D). Furthermore, we found unique molecular profiles for the majority of MNs. In A1R alone we identified 23 subtypes of MNs (Fig. 8E; supplementary material Table S3 and Data File S8), whereas previously only seven molecularly distinct MN subtypes were known (Landgraf and Thor, 2006). We conclude that the eNeuro atlas (1) accurately identifies all known MNs and midline cells; (2) reveals unprecedented molecular diversity in MNs and midline cells; and (3) provides novel tools for studying cells that were previously genetically inaccessible (see Discussion).

\section{DISCUSSION}

To our knowledge, atlases generated with our 'atlas-builder' software are unique in allowing the integration of an unlimited number of new markers. For example, the Allen Brain Atlas has an infinitely expandable number of pages for gene expression profiles, but the expression patterns are not linked, and it is unclear which fine structures co-express different markers. In contrast, we use a single fiduciary marker to register new patterns to the existing atlas; thus, an unlimited number of markers can be co-stained with the fiduciary marker, added to the atlas and their overlap predicted with high accuracy. A second important feature of an atlas generated with our software is that users can perform a 'BLAST'-like search to predict which known cells express a new marker. Although perfect accuracy is probably impossible due to existing biological variation, the ranking of nearby candidate cells will greatly facilitate identification of cells that express a new marker. Our tools are publicly available, and we provide detailed instructions to guide new users in creating and 'BLASTing' atlases for new markers (supplementary material Data File S9).

Previously, only a handful of Drosophila embryonic interneurons were individually identifiable using a combination of molecular marker expression and cell body position - e.g. pCC, EW1-EW3, Apterous+ neurons and Eve+ lateral interneurons (Doe et al., 1988; Higashijima et al., 1996; Park et al., 2004; Thomas et al., 1984; Tsuji et al., 2008). The eNeuro atlas expands the number of identified interneurons to 958, each of which has been assigned a unique name, and each of which is described by quantitative information on cell body position, size and frequency of appearance, as well as its Gal4 line expression profile (supplementary material Data File S8). Nevertheless, the eNeuro atlas is not yet 'saturated' for interneurons: $\sim 40 \%$ of interneurons do not express any marker (Fig. 5). Importantly, it is simple to add new markers to the eNeuro atlas (see below). We anticipate that additional markers will allow the unique identification of every interneuron in the ventral CNS. In the future, we will provide updated marker atlas files and add atlas 


\begin{tabular}{|c|c|c|c|c|c|c|}
\hline$\infty \rightarrow \infty$ & $\infty$ & , & 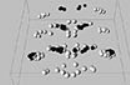 & . & $\therefore$ & . \\
\hline A227 & R10D02 & R10D07 & R11F02 & R11F04 & $\mathrm{R} 12 \mathrm{~A} 10$ & R12B12 \\
\hline$\therefore$ & : & $=0$ & 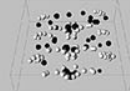 & 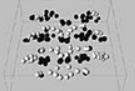 & 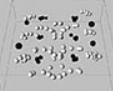 & 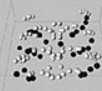 \\
\hline R12E03 & R12E07 & R13D08 & R13E06 & R14E12 & $\mathrm{R} 14 \mathrm{H} 08$ & R15D07 \\
\hline$\sum_{\infty}^{-\infty}$ & $=\infty$ & 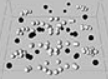 & 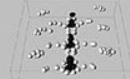 & wo & 20 & 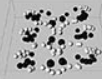 \\
\hline R15E11 & R15F01 & R17C08 & R17F04 & R18A04 & R19A07 & $\mathrm{R} 19 \mathrm{H} 09$ \\
\hline$\$ 0$ & . & $\infty$ & 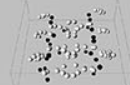 & 等, & 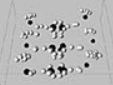 & 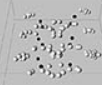 \\
\hline R20A12 & $\mathrm{R} 21 \mathrm{~A} 04$ & R21B12 & $\mathrm{R} 21 \mathrm{C} 06$ & $\mathrm{R} 22 \mathrm{C} 10$ & $\mathrm{R} 23 \mathrm{~A} 02$ & R23G10 \\
\hline 等 & 烈 & : & - & $\therefore$ & 蓶 & 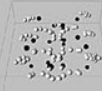 \\
\hline R23G11 & $\mathrm{R} 25 \mathrm{CO} 3$ & R25D09 & $\mathrm{R} 25 \mathrm{G} 03$ & $\mathrm{R} 25 \mathrm{H} 11$ & R26B03 & $\mathrm{R} 26 \mathrm{H} 02$ \\
\hline 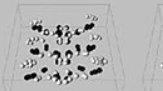 & . & , & . & and & 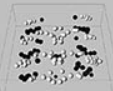 & $\therefore$ \\
\hline R27A09 & R29A12 & $\mathrm{R} 30 \mathrm{~A} 02$ & R30E03 & R31G06 & R33E02 & R33G07 \\
\hline 舟, & : & 数: & $\Leftrightarrow 3$ & 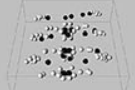 & 30 & 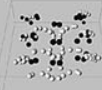 \\
\hline R34D08 & R35D09 & R35D11 & R37G07 & R38C08 & R38E07 & $\mathrm{R} 45 \mathrm{D} 07$ \\
\hline 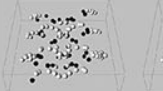 & 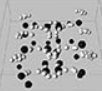 & ? & 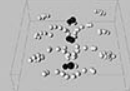 & . & 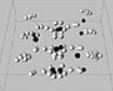 & , \\
\hline R46A06 & $\mathrm{R} 47 \mathrm{H} 04$ & R48G05 & R50B02 & R52B03 & R53B03 & R53E03 \\
\hline 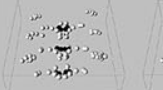 & , & $\therefore$ & 80 & 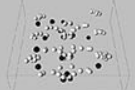 & $\infty$ & $\rightarrow 0$ \\
\hline R53F07 & R54B03 & R54E06 & R54E08 & $\mathrm{R} 55 \mathrm{C} 06$ & R55E04 & R59D01 \\
\hline : & - & 争, & के & (1) & 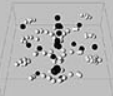 & to \\
\hline R64A12 & R64B05 & R64B06 & R65B10 & R65D03 & $\mathrm{R} 65 \mathrm{H} 11$ & $\mathrm{R} 66 \mathrm{C} 01$ \\
\hline 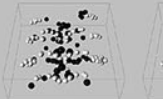 & $\because$ & 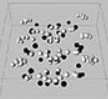 & $=$ & tho & & \\
\hline R71D07 & $\mathrm{R} 71 \mathrm{~F} 10$ & R74B12 & R75G10 & $9-58$ & & \\
\hline \multirow[b]{2}{*}{$\begin{array}{l}\text { average stdev } \\
\text { (cell diameters) }\end{array}$} & $0_{1}^{0}$ & 400 & 300,800 & 1000,12 & \multicolumn{2}{|c|}{ number of cells } \\
\hline & s) $\frac{0-0.5}{\text { more }}$ & & & & \multicolumn{2}{|l|}{$65 \%$} \\
\hline
\end{tabular}

Fig. 4. Single-marker atlases for $75 \mathrm{Gal} 4$ lines expressed in small subsets of neurons. Single-marker atlases for $75 \mathrm{Gal} 4$ lines. Gal4+ nuclei are shown in black, with the name underneath; Eve+ nuclei are white; anterior is upwards; ventral view with $45^{\circ}$ rotation about the anterior-posterior axis. For a list of all individual cells and statistics, see supplementary material Data File S5. Histogram showing the distribution of average s.d. of nuclear position for cells labeled by Gal4 lines. files from other users with their permission. In addition, we plan to add markers that identify individual neuroblast lineages.

Until now, few genetic tools existed to manipulate specific subtypes of embryonic interneurons. Here, we have mapped 75 Gal4 lines that each label small, reproducible subsets of embryonic interneurons (Fig. 4). Some lines show the same pattern in larvae while others change pattern over time (data not shown). Importantly, the use of 'immortalization' genetics (Buckingham and Meilhac, 2011) can make many of these Gal4 markers useful at later stages of development or in the adult. Furthermore, many interneurons in the eNeuro atlas were labeled by multiple Gal4 lines (Fig. 6A,C; supplementary material Fig. S1), thereby raising the possibility of using intersectional genetics to target single interneurons, because the vast majority of these lines were made with a defined cis-regulatory DNA fragment (Pfeiffer et al., 2010). Thus, this work provides a significant advance in our ability to characterize and test the function of individual identified interneurons.

We also provide important new tools for the study of MNs and midline cells (Figs 7,8; supplementary material Tables S2,S3). These can be used to trace the axon projection of a previously uncharacterized Eve/Hb9/Bar triple-negative motor neuron (supplementary material Fig. S2). In the future they could be used to study the specification, axon pathfinding, target recognition and role in motor behavior. We also made a novel discovery regarding the well-studied $\mathrm{Hb} 9+\mathrm{MNs}$ : although most $\mathrm{Hb} 9+\mathrm{MNs}$ project out of the intersegmental nerve (ISN), some have been reported to project out of the segmental nerve root $(\mathrm{SN})$ to transverse somatic muscles, similar to the projection pattern of the Bar+ MNs (Broihier 


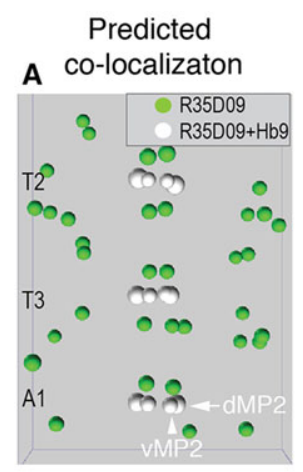

C

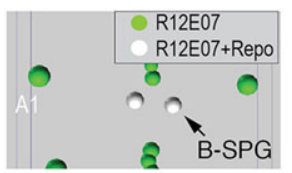

In vivo validated co-localization
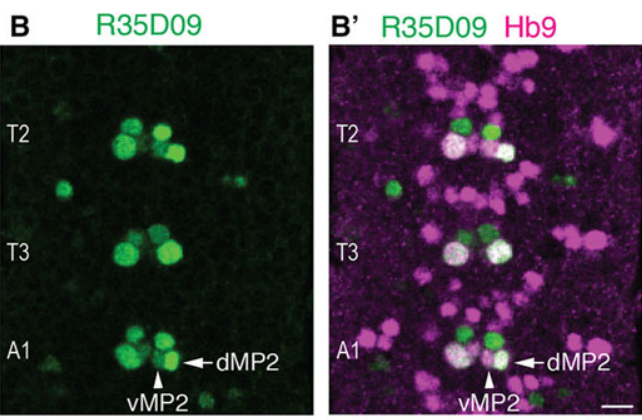

D R12E07

(nuclear)

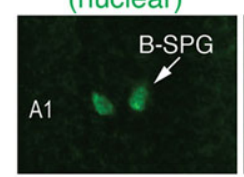

D' R12E07

Repo

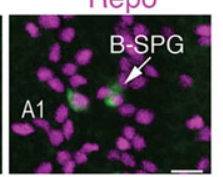

E R12E07 (membrane)

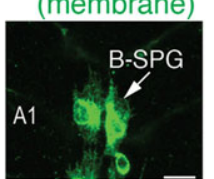

Fig. 5. Single marker atlases can be unified in atlases. $\left(A, B^{\prime}\right)$ Atlaspredicted and in vivo-validated co-expression (A) R35D09 and Hb9 are predicted to co-express (white) in dMP2 (arrowhead) and VMP2 (arrow). (B, $\mathrm{B}^{\prime}$ ) In vivo validation of $\mathrm{R} 35 \mathrm{D} 09$ and $\mathrm{Hb} 9$ co-expression in dMP2 and vMP2. Anterior is upwards. Scale bar: $10 \mu \mathrm{m}$. (C-E) Atlaspredicted and in vivo-validated co-expression. (C) R12E07 and Repo are predicted to co-express (white) in the B-SPG (arrow) glial cell. $\left(\mathrm{D}, \mathrm{D}^{\prime}\right)$ In vivo validation of $\mathrm{R} 12 \mathrm{E} 07$ and Repo co-expression in B-SPG. (E) R12E07-driving membrane GFP reveals the characteristic subperineural glia morphology in the B-SPG. Scale bars: $10 \mu \mathrm{m}$. Anterior is upwards. and Skeath, 2002; Garces et al., 2006). Our atlas did not contain any Hb9+Bar+ co-expressing neurons, which was confirmed by experiments showing no $\mathrm{Hb} 9+$ neurons projecting out of the $\mathrm{SN}$ to transverse muscles (supplementary material Fig. S3). We conclude that the $\mathrm{Hb} 9+\mathrm{MNs}$ do not co-express Bar or project out of the SN, at least not at embryonic stage 16. Thus, the eNeuro atlas provides tools for the developmental and functional analysis of individual and poorly characterized groups of MNs.

Limitations of the atlas approach include potential annotation errors that exaggerate positional variability, and stochasticity in Gal4 expression that results in some cells being unrepresented or unilaterally represented. It is also possible that the eNeuro atlas contains errors in cell merging. Errors are likely to be rare, however, because we examined 171 of 243 predicted Gal4/TF co-expressing cells and found only one error, which was manually corrected (supplementary material Data File S5); the remaining Gal4/Gal4 merge predictions are likely to have a similarly high accuracy.

The genetic analysis of neural development in Drosophila has led to many fundamental discoveries that also apply to mammalian neural development. The study of interneuron specification and
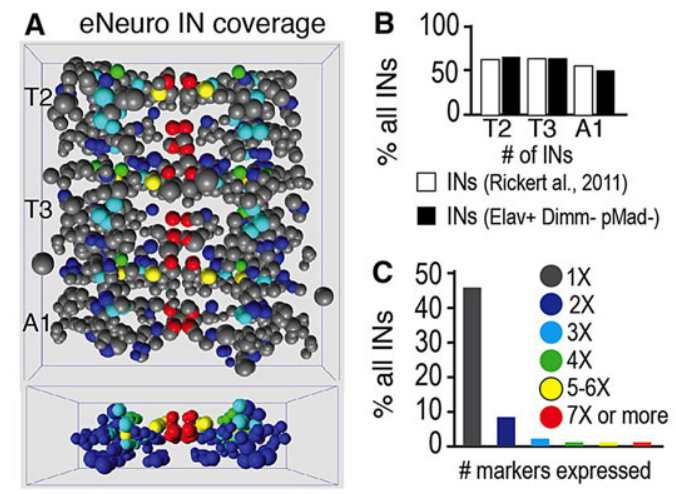

Fig. 6. eNeuro atlas labels a majority of interneurons. (A) All interneurons in the eNeuro atlas. Each neuron is color-coded according to the number of markers it expresses: 1 (gray), 2 (blue), 3 (teal), 4 (green), 5-6 (yellow), $>6$ (red). Top panel, anterior is upwards; bottom panel, dorsal is upwards. (B) eNeuro atlas identifies a majority of interneurons in the ventral CNS. White bars indicate coverage based on 270 interneurons per hemisegment (Rickert et al., 2011). Black bars indicate coverage based on total number of Elav+pMad-Dimm- neurons (see Materials and Methods). (C) A fraction of the interneurons in the eNeuro atlas is multiply labeled. Colors as in A. function is in its infancy in both Drosophila and mammals. We suggest that this atlas of interneuron diversity and tools for functional analysis will facilitate the study of Drosophila interneurons, and that this will have a direct impact on the study of mammalian interneurons. This is an important area of research, as interneuron dysfunction is thought to underlie autism, epilepsy and other debilitating human neural disorders.
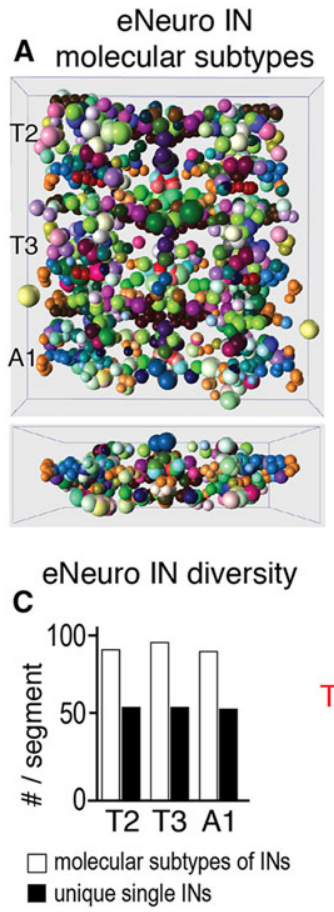

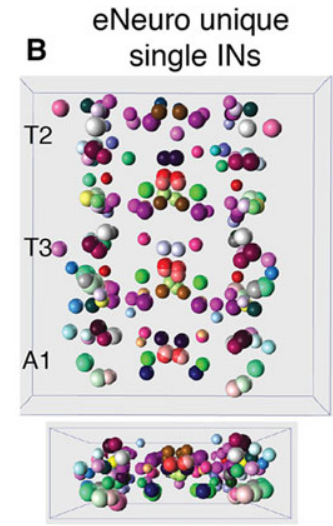

112 molecular

D subtypes of INs

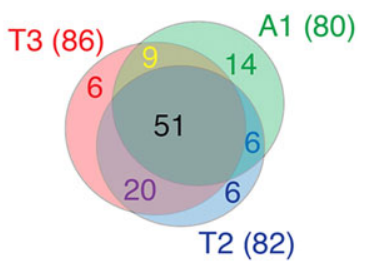

Fig. 7. eNeuro atlas subdivides interneurons into 112 distinct molecular subtypes. (A) All interneurons included in the eNeuro atlas. Each molecularly different subtype is represented by a different color (supplementary material Data File S8 and Table S1). Top panel, anterior is upwards; bottom panel, dorsal is upwards. (B) The subset of interneurons (from $6 \mathrm{~A}$ ) for which a single neuron in each hemisegment has a unique molecular profile. View as in $A$. (C) eNeuro atlas reveals interneuron molecular diversity. White bars indicate molecularly distinct interneuron subtypes (in A). Black bars indicate molecularly unique single interneurons (in B). (D) There are 112 distinct molecular subtypes in the eNeuro atlas: 80 subtypes in A1 (green), 82 in T2 (purple) and 86 in T3 (red). 

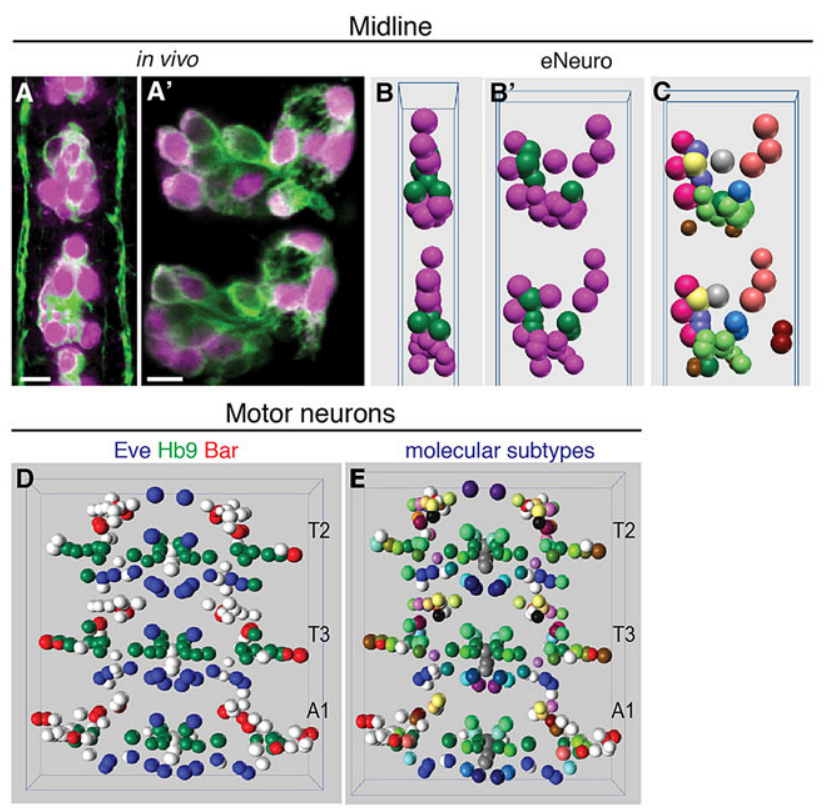

Fig. 8. Midline and motor neuron molecular diversity in the eNeuro atlas. $\left(A, A^{\prime}\right)$ Composite image of a stage 16 sim-Gal4 UAS-tau-GFP embryo stained with anti-GFP (green) and anti-Sim (magenta). ( $\left.A^{\prime}\right)$ Sagittal view. $\left(B, B^{\prime}\right)$ Atlas view of Sim+ (magenta) and Sim - (green) midline cells. (B) Dorsal view; anterior is upwards. View as in $A, A^{\prime}$. (C) Atlas sagittal view of midline cells with each subtype represented as a different color (supplementary material Table S3). (D) pMad+ MNs subdivided in four molecular subtypes: Bar+pMad+ cells (red); Hb9+pMad+ (green) cells; Eve+pMad+ (blue); and poorly characterized triple-negative pMad+ (white) MNs. There is no overlap between $\mathrm{Hb} 9+$, Bar+ and Eve+ cells. Anterior is upwards. (e) The eNeuro atlas increases the number of molecularly distinct motor neuron subtypes in A1-23, each of which indicated by a different color (supplementary material Table S3). Anterior is upwards.

\section{MATERIALS AND METHODS \\ Fly genetics}

We used standard methods for propagating fly stocks. Transcription factor patterns were determined in wild-type flies (yellow white). Gal4 patterns were determined by crossing Gal4 [BN-gal4 (Sato et al., 1999), Ap-Gal4 (Bloomington \#3041); 9-58-Gal4 (Ulrike Heberlein, Janelia Farm Research Campus, JFRC); a227-Gal4 (Julie Simpson, JFRC); Hb9-Gal4 (Broihier and Skeath, 2002); and all other Gal4s (JRFC) (Pfeiffer et al., 2008)] males to females containing $U A S-G F P$ with a nuclear localization sequence or membrane tether (Bloomington \#6452, \#32184, \#32197).

\section{Embryo immunostaining and staging}

We used standard methods to stain Drosophila embryos (Manning et al., 2012). Primary antibodies were mouse Eve 2B8 (1:50), mouse En 4D9 (1:10), and rat Elav (1:10), all from the Developmental Studies Hybridoma Bank (DSHB) developed under the National Institute of Child Health and Human Development (NICHD) and maintained by the University of Iowa; guinea pig Dimm (1:100; a gift from S. Thor, Linkoping University, Sweden); rat Repo (Campbell et al., 1994) (1:1000); rabbit pMad (pS1 1:300; Peter Ten Dijke, Leiden University Medical Center, Leiden, The Netherlands); chicken GFP (1:1000; Aves, Tigard, OR); rabbit Hb9 (Odden et al., 2002) (1:1000); and guinea pig Fox (Nechipurenko and Broihier, 2012) (1:20). Secondary antibodies were from Invitrogen/Molecular Probes and were used according to the manufacturer's instructions. Stage 16 embryos were selected for imaging based on morphological criteria (Pereanu et al., 2007).

\section{Image acquisition}

Images were acquired on a BioRad Radiance, Zeiss 700 or 710 confocal microscope with a $63 \times$ objective collected at 0.3 or $0.5 \mu \mathrm{m}$ steps. Images were cropped in ImageJ (NIH) to include segments T2-A1. Some images were used to generate atlases, whereas others were used to validate co-expression or for figures.

\section{Segmentation}

We developed a Vaa3D (Peng et al., 2010) (http://Vaa3d.org/) plug-in to segment cells. First, a TIFF image was smoothed with a median filter and/or Gaussian filter to reduce noise. We then separated the image into background and foreground pixels to make the later segmentation step more robust and computationally efficient. We provided three thresholding methods: adaptive thresholding, global 3D thresholding and global 2D thresholding. After thresholding, we used a watershed algorithm to segment cells based on either the intensity information or the shape information of foreground pixels. For each transcription factor we segmented and annotated a median of 10 image stacks. We segmented a median of five image stacks per Gal4 line.

\section{Annotation}

The segmented cells were annotated using VANO (Peng et al., 2009) software (http://vano.cellexplorer.org/). Where possible, neuron names were taken from the literature and had the following syntax: "name/marker hemisegment' (e.g. pCC/eve T2R or A/19H09 T2R). Segment identity was based on neuron position at mid stage 16; the U1-U5 neurons defined the posterior boundary of each segment. Ruby scripts were used to detect annotation errors, such as typos (code is available upon request). To minimize annotation, we used only Eve+ medial neurons (aCC, pCC, RP2, U1-U5) in each segment, as this was sufficient for optimal registration (data not shown). For new markers, annotation could be carried out on just one cell of interest or on all cells.

\section{Registration}

A newly developed Vaa3D plug-in called 'AtlasBuilder' uses rigid registration to map the annotated image stacks into a standard coordinate space defined by a target stack. The target stack was automatically determined from a randomly selected subset of stacks as the one that minimized the summed cell position variations (supplementary material Data File S1). We computed an affine transformation that best aligned the EVE channel of a subject stack to that of the target stack. This transformation was then applied to all channels of an image stack. Note that this type of rigid registration does not use local warping to align the neurons. It calculates an average position for each nucleus, which acts as unique $x, y, z$ coordinates in virtual space; it also calculates the s.d. of the variance of actual centers from the mean, which acts as a measure of the stereotypy of each cell. For a complete list of data produced by registration, see the 'cellStatistics.xls' file for each atlas (supplementary material Data Files S2, S3 and S5). 'AtlasBuilder' can build atlases with different confidence thresholds, which are calculated based on how often a given cell appears in image stacks. For transcription factor atlases, we included only cells that were found in $33 \%$ or more of the image stacks; for Gal4 atlases, we included all cells.

\section{Co-expression prediction and confirmation}

To establish in vivo co-expression between markers, we used data from previously published reports, or performed co-labeling experiments ourselves. We determined the individual identity of each cell based on the relative position of the cell within the marker pattern, as we did for manual annotation. We also scored for projection patterns/cell morphology and for nuclear shape where these characteristics had been reported in the literature (supplementary material Data File S5).

To predict overlap between single marker atlases, we developed a 'Detect coexpressing cells' function in AtlasBuilder. This method takes '.apo' files (single atlases and/or atlases) and determines which cells are within a userspecified distance (we used six pixels, $\sim$ average nuclear radius). We did not use symmetry or bilateral rules, although they are available (supplementary material Data File S9 for details). This plug-in generates two output files: a '. coexpress.apo' where predicted co-expression is white, and a '. coexpress. txt' file, which lists each candidate cell followed by all cells found within the specified radius. 
To calculate prediction accuracy we calculated three parameters: 'true positives' - co-expression found both by atlas prediction and in vivo analysis; 'false negatives' - co-expression found only by in vivo analysis; 'false positive' - co-expression found only by atlas prediction. True positives and false negatives were scored as a percentage of all expected $(n=133)$ in vivo co-expressions (supplementary material Data File S5). False positives were scored as a percentage of total predictions.

We used the following three rules.

Rule 1: if a cell in one hemisegment is predicted to co-express, then all examples of that cell also co-express; $96 \%$ true positive rate, $4 \%$ false negative rate and $51 \%$ false positive rate.

Rule 2: if the same cell in two hemisegments is predicted to co-express, then all examples of that cell also co-express; $84 \%$ true positives, $16 \%$ false negatives and $30 \%$ false positives.

Rule 3: if the same cell in three hemisegments is predicted to co-express, then all examples of that cell also co-express; $40 \%$ true positives, $60 \%$ false negatives and $6 \%$ false positives.

\section{Merging}

The 'Merge coexpressing cells' plug-in for Vaa3d 'AtlasBuilder' takes two types of input: 'apo' files and a user-generated look-up table text file (supplementary material Data File S10). The merging plug-in produces a new atlas with both '.apo' and 'cellstatistics.txt' files containing positional information for the new cells; these new positions are calculated from the average positions of all original cells, even if they come from two separate '.apo' files. In the resulting atlas, all markers expressing in a given cell are listed in the comments column of the '.apo' files.

To create the TF atlas, we assigned each cell a new name, which was a truncated version of the original names (i.e. aCC/eve T2R and aCC/pMad T2R became aCC T2R)

To create the eNeuro atlas, we discarded any low-quality cells: cells appearing in less than $33 \%$ of the annotated Gal4 line image stacks, or cells with an average s.d. of the nuclear position that was greater than a single cell diameter. We used the 'Detect coexpressing cells' function to generate a list of predicted co-expressions among all 83 single atlases. We used custom Ruby scripts to apply 'Rule 2' (see above). We then used Ruby scripts and MATLAB to view the matrix of co-expression as biograph objects (code is available upon request). We merged cells in the following order: MPs (dMP2, vMP2, MP1), Eve+ cells, Bar+ cells, Hb9+ cells, pMad + cells, cells predicted to label with other transcription factors, all other cells. Each subtype was assigned a name; if the cell appeared in the TF atlas, we used the same name; all others ( 646 cells) were assigned names using 'IN' as a prefix followed by a three-digit number, such as 'IN094' (see supplementary material Data File S10 for complete list of merged cells).

\section{Other calculations}

Cell diameter was calculated using the mean volume of all cells and then solved for $\mathrm{r}\left(\right.$ volume $\left.=4 / 3 \pi \mathrm{r}^{3}\right)$. The s.d. of cell position was calculated as $x, y, z$ s.d. (std_x, std_y, std_z)/3 in Fig. 1. In Figs 2 and 4, average s.d. was calculated similarly, but we combined data from cell bodies in all hemisegments (e.g. aCC represents data from aCC/eve T2R, T2L, T3R, T3L, A1R and A1L).

We used two methods to estimate the number of interneurons in each segment: 540 interneurons, based on single interneuron DiI labeling (Campbell et al., 1994), which does not include unpaired midline neurons; or an average of 549 interneurons per segment (515 in T2, 542 in T3, 589 in A1), based on our own counts of Elav+ neurons minus pMad+ MNs and Dimm+ neurosecretory cells. To determine the interneuron coverage in the eNeuro atlas, we defined interneurons as triple negative for pMad (MNs), Dimm (neurosecretory cells) and Repo (medial glia), as well as having low positional variability (thus excluding lateral glia). This gave us an average of 324 interneurons per segment (Fig. 6; 337 in T2, 339 in T3, 295 in A1).

\section{Acknowledgements}

We thank Matt Clark, Sen-Lin Lai and Chris Wreden for comments on the manuscript; Maria Purice, Art Li, Andrew Reichold and Jimmy Kelly for help annotating marker patterns; and the Drosophila Stock Center (Bloomington, IN, USA) and Todd Laverty at the JFRC (VA, USA) for fly stocks.

\section{Competing interests}

The authors declare no competing financial interests.

\section{Author contributions}

M.J.L. and F.L. initiated the project; F.L., H.P. and E.M. developed software; J.C.P. and S.T.C. tested software prior to publication and analyzed midline cells; E.S.H., M.J.L., C.-H.C., L.M. and J.R. contributed to data acquisition, image processing, annotation and registration; E.S.H. merged atlases and performed data analysis; E.S.H. and C.Q.D. wrote the manuscript.

\section{Funding}

This work was funded by the American Heart Association [\#0920025G postdoctoral fellowship to E.S.H.], the National Institutes of Health [27056 to C.Q.D.], the Howard Hughes Medical Institute (C.Q.D.), the National Institute of Neurological Disorders and Stroke [R01 NS64264 to S.T.C.] and the JFRC (F.L., H.P. and E.M.). Deposited in PMC for release after 12 months.

\section{Supplementary material}

Supplementary material available online at

http://dev.biologists.org/lookup/suppl/doi:10.1242/dev.108720/-/DC1

\section{References}

Alaynick, W. A., Jessell, T. M. and Pfaff, S. L. (2011). SnapShot: spinal cord development. Cell 146, 178-178. e1.

Allan, D., Pierre, S., Miguel-Aliaga, I. and Thor, S. (2003). Specification of neuropeptide cell identity by the integration of retrograde BMP signaling and a combinatorial transcription factor code. Cell 113, 73-86.

Arber, S. (2012). Motor circuits in action: specification, connectivity, and function Neuron 74, 975-989.

Beckervordersandforth, R. M., Rickert, C., Altenhein, B. and Technau, G. M. (2008). Subtypes of glial cells in the Drosophila embryonic ventral nerve cord as related to lineage and gene expression. Mech. Dev. 125, 542-557.

Benveniste, R. J., Thor, S., Thomas, J. B. and Taghert, P. H. (1998). Cell typespecific regulation of the Drosophila FMRF-NH2 neuropeptide gene by Apterous, a LIM homeodomain transcription factor. Development 125, 4757-4765.

Bourgouin, C., Lundgren, S. and Thomas, J. (1992). apterous is a Drosophila LIM domain gene required for the development of a subset of embryonic muscles. Neuron 9, 549-561.

Broadus, J., Skeath, J. B., Spana, E. P., Bossing, T., Technau, G. and Doe, C. Q. (1995). New neuroblast markers and the origin of the aCC/pCC neurons in the Drosophila central nervous system. Mech. Dev. 53, 393-402.

Broihier, H. T. and Skeath, J. B. (2002). Drosophila homeodomain protein dHb9 directs neuronal fate via crossrepressive and cell-nonautonomous mechanisms. Neuron 35, 39-50.

Buckingham, M. E. and Meilhac, S. M. (2011). Tracing cells for tracking cell lineage and clonal behavior. Dev. Cell 21, 394-409.

Campbell, G., Göring, H., Lin, T., Spana, E., Andersson, S., Doe, C. Q. and Tomlinson, A. (1994). RK2, a glial-specific homeodomain protein required for embryonic nerve cord condensation and viability in Drosophila. Development 120 , 2957-2966.

Chiang, C., Patel, N. H., Young, K. E. and Beachy, P. A. (1994). The novel homeodomain gene buttonless specifies differentiation and axonal guidance functions of Drosophila dorsal median cells. Development 120, 3581-3593.

Diez-Roux, G., Banfi, S., Sultan, M., Geffers, L., Anand, S., Rozado, D., Magen, A., Canidio, E., Pagani, M., Peluso, I. et al. (2011). A high-resolution anatomical atlas of the transcriptome in the mouse embryo. PLoS Biol. 9, e1000582.

Doe, C. Q., Smouse, D. and Goodman, C. S. (1988). Control of neuronal fate by the Drosophila segmentation gene even-skipped. Nature 333, 376-378.

Frasch, M., Hoey, T., Rushlow, C., Doyle, H. and Levine, M. (1987) Characterization and localization of the even-skipped protein of Drosophila. EMBO J. 6, 749-759.

Fujioka, M., Emi-Sarker, Y., Yusibova, G. L., Goto, T. and Jaynes, J. B. (1999). Analysis of an even-skipped rescue transgene reveals both composite and discrete neuronal and early blastoderm enhancers, and multi-stripe positioning by gap gene repressor gradients. Development 126, 2527-2538.

Garces, A., Bogdanik, L., Thor, S. and Carroll, P. (2006). Expression of DrosophilaBarH1-H2 homeoproteins in developing dopaminergic cells and segmental nerve a (SNa) motoneurons. Eur. J. Neurosci. 24, 37-44.

Higashijima, S., Shishido, E., Matsuzaki, M. and Saigo, K. (1996). eagle, a member of the steroid receptor gene superfamily, is expressed in a subset of neuroblasts and regulates the fate of their putative progeny in the Drosophila CNS. Development 122, 527-536.

Ito, K., Urban, J. and Technau, G. (1995). Distribution, classification, and development of Drosophila glial cells in the late embryonic and early larval ventral nerve cord. Dev. Genes Evol. 204, 284-307.

Kearney, J. B., Wheeler, S. R., Estes, P., Parente, B. and Crews, S. T. (2004) Gene expression profiling of the developing Drosophila CNS midline cells. Dev. Biol. 275, 473-492. 
Landgraf, M. and Thor, S. (2006). Development of Drosophila motoneurons: specification and morphology. Semin. Cell Dev. Biol. 17, 3-11.

Landgraf, M., Bossing, T., Technau, G. M. and Bate, M. (1997). The origin location, and projections of the embryonic abdominal motorneurons of Drosophila. J. Neurosci. 17, 9642-9655.

Layden, M. J., Odden, J. P., Schmid, A., Garces, A., Thor, S. and Doe, C. Q. (2006). Zfh1, a somatic motor neuron transcription factor, regulates axon exit from the CNS. Dev. Biol. 291, 253-263.

Lein, E. S., Hawrylycz, M. J., Ao, N., Ayres, M., Bensinger, A., Bernard, A., Boe, A. F., Boguski, M. S., Brockway, K. S., Byrnes, E. J. et al. (2007). Genomewide atlas of gene expression in the adult mouse brain. Nature 445, 168-176.

Lundgren, S., Callahan, C., Thor, S. and Thomas, J. (1995). Control of neuronal pathway selection by the Drosophila LIM homeodomain gene apterous. Development. 121, 1769-1773.

Manning, L., Heckscher, E. S., Purice, M. D., Roberts, J., Bennett, A. L., Kroll, J. R. Pollard, J. L., Strader, M. E., Lupton, J. R., Dyukareva, A. V. et al. (2012). A resource for manipulating gene expression and analyzing cis-regulatory modules in the Drosophila CNS. Cell Rep. 2, 1002-1013.

Mccabe, B. D., Marqués, G., Haghighi, A. P., Fetter, R. D., Crotty, M. L., Haerry, T. E., Goodman, C. S. and O'Connor, M. B. (2003). The BMP homolog Gbb provides a retrograde signal that regulates synaptic growth at the Drosophila neuromuscular junction. Neuron 39, 241-254.

Miguel-Aliaga, I. (2004). Independent roles of the dachshund and eyes absent genes in BMP signaling, axon pathfinding and neuronal specification. Development 131, 5837-5848.

Miguel-Aliaga, I., Thor, S. and Gould, A. (2008). Postmitotic specification of Drosophila insulinergic neurons from pioneer neurons. PLoS Biol. 6, e58.

Nechipurenko, I. V. and Broihier, H. T. (2012). FoxO limits microtubule stability and is itself negatively regulated by microtubule disruption. J. Cell Biol. 196, 345-362.

Odden, J. P., Holbrook, S. and Doe, C. Q. (2002). Drosophila HB9 is expressed in a subset of motoneurons and interneurons, where it regulates gene expression and axon pathfinding. J. Neurosci. 22, 9143-9149.

O'Connor, M. B. (2005). Shaping BMP morphogen gradients in the Drosophila embryo and pupal wing. Development 133, 183-193.

Park, D., Han, M., Kim, Y.-C., Han, K.-A. and Taghert, P. H. (2004). Ap-let neurons-a peptidergic circuit potentially controlling ecdysial behavior in Drosophila. Dev. Biol. 269, 95-108.

Park, D., Veenstra, J. A., Park, J. H. and Taghert, P. H. (2008). Mapping peptidergic cells in Drosophila: where DIMM fits in. PLOS ONE 3, e1896.

Patel, N. H., Martin-Blanco, E., Coleman, K. G., Poole, S. J., Ellis, M. C., Kornberg, T. B. and Goodman, C. S. (1989). Expression of engrailed proteins in arthropods, annelids, and chordates. Cell 58, 955-968.

Peng, H., Long, F. and Myers, E. W. (2009). VANO: a volume-object image annotation system. Bioinformatics 25, 695-697.
Peng, H., Ruan, Z., Long, F., Simpson, J. H. and Myers, E. W. (2010). V3D enables real-time $3 D$ visualization and quantitative analysis of large-scale biological image data sets. Nat. Biotechnol. 28, 348-353.

Pereanu, W., Spindler, S., Im, E., Buu, N. and Hartenstein, V. (2007). The emergence of patterned movement during late embryogenesis of Drosophila. Devel. Neurobio. 67, 1669-1685.

Pfeiffer, B. D., Jenett, A., Hammonds, A. S., Ngo, T.-T. B., Misra, S., Murphy, C., Scully, A., Carlson, J. W., Wan, K. H., Laverty, T. R. et al. (2008). Tools for neuroanatomy and neurogenetics in Drosophila. Proc. Natl. Acad. Sci. U.S.A. 105, 9715-9720.

Pfeiffer, B. D., Ngo, T.-T. B., Hibbard, K. L., Murphy, C., Jenett, A., Truman, J. W. and Rubin, G. M. (2010). Refinement of tools for targeted gene expression in Drosophila. Genetics 186, 735-755.

Rickert, C., Kunz, T., Harris, K.-L., Whitington, P. M. and Technau, G. M. (2011) Morphological characterization of the entire interneuron population reveals principles of neuromere organization in the ventral nerve cord of Drosophila. J. Neurosci. 31, 15870-15883.

Robinow, S. and White, K. (1988). The locus elav of Drosophila melanogaster is expressed in neurons at all developmental stages. Dev. Biol. 126, 294-303.

Sato, M., Kojima, T., Michiue, T. and Saigo, K. (1999). Bar homeobox genes are latitudinal prepattern genes in the developing Drosophila notum whose expression is regulated by the concerted functions of decapentaplegic and wingless. Development 126, 1457-1466.

Schmid, A., Chiba, A. and Doe, C. (1999). Clonal analysis of Drosophila embryonic neuroblasts: neural cell types, axon projections and muscle targets. Development.

Thomas, J. B., Bastiani, M. J., Bate, M. and Goodman, C. S. (1984). From grasshopper to Drosophila: a common plan for neuronal development. Nature 310, 203-207

Tomancak, P., Berman, B. P., Beaton, A., Weiszmann, R., Kwan, E., Hartenstein, V., Celniker, S. E. and Rubin, G. M. (2007). Global analysis of patterns of gene expression during Drosophila embryogenesis. Genome Biol. 8 , R145.

Tsuji, T., Hasegawa, E. and Isshiki, T. (2008). Neuroblast entry into quiescence is regulated intrinsically by the combined action of spatial Hox proteins and temporal identity factors. Development 135, 3859-3869.

Wheeler, S. R., Kearney, J. B., Guardiola, A. R. and Crews, S. T. (2006). Singlecell mapping of neural and glial gene expression in the developing Drosophila CNS midline cells. Dev. Biol. 294, 509-524.

Wheeler, S. R., Stagg, S. B. and Crews, S. T. (2009). MidExDB: a database of Drosophila CNS midline cell gene expression. BMC Dev. Biol. 9, 56.

Xiong, W. C., Okano, H., Patel, N. H., Blendy, J. and Montell, C. (1994). repo encodes a glial-specific homeo domain protein required in the Drosophila nervous system. Genes Dev. 8, 981-994. 